\title{
Reliability-Based Design Optimization of RC Frames Based on the RBDO-Kriging Method
}

\author{
Guitao Chen ${ }^{1,2, *}$ and Demin Wei ${ }^{1}$ \\ ${ }^{1}$ School of Civil Engineering and Transportation, South China University of Technology, Guangzhou, China \\ ${ }^{2}$ Shenzhen Institute of Building Research Co.Ltd, Shenzhen, China \\ *Corresponding author
}

\begin{abstract}
Reliability-based design optimization (RBDO) is a more rational optimization design method due to its consideration of the structure reliability requirements. A new RBDO-kriging method that combines kriging agent model and covariance matrix adaptation evolution strategy (CMAES) is proposed to solve the problems of RBDO of concrete frames efficiently. RBDO of a ten-story and two-bay concrete frame is implemented, the results obtained by comparing five working conditions show that, the refining kriging model proposed in this paper is effective, and RBDO-kriging algorithm can obtain better solution than deterministic optimization algorithm and optimization algorithm based on basic kriging model.
\end{abstract}

Keywords- RBDO-kriging algorithm; kriging model; covariance matrix adaptation evolution strategy; reliability-based design optimization

\section{INTRODUCTION}

In order to deal with this kind of uncertainties in structural optimization design, the reliabilities should be imported in the optimization design models [1-3], which is named as reliability based design optimization. There are three algorithms to solve the RBDO problems. The double loop algorithm which nests the reliability calculation loop in the optimization loop is the most direct approach. Recently, scholars have developed some technologies for the effective RBDO problem solving [7-11], such as the approximate reliability, advanced simulation, met model, High Performance Computing and agent model etc. The agent model is a simulative model to replace the structural analysis model, which can reduce computing burden, smooth the numerical noise and result in the prevalence of the agent model when tackling the RBDO problem [9, 12-14].

To this end, combining the computing advantages of kriging agent model and covariance matrix adaptation evolution strategy (CMAES), this paper proposes a new RBDO-kriging method to solve the problem of RBDO of concrete frames.

\section{RBDO-KRIGING MODEL}

RBDO formula.RBDO formula can be written as:

$\min : \mathrm{C}(\mathbf{d})$ s.t.: $\beta_{i}(\mathbf{d}, \mathbf{X}) \geq \beta_{i}{ }^{T}$

$\mathrm{i}=1, \cdots, \mathrm{m} ; h_{j}(\mathrm{~d}) \leqslant 0 ; \mathrm{j}=\mathrm{m}+1, \cdots, \mathrm{M}$

where, $\mathbf{d}$ is the design variable vector; $\mathbf{X}$ is the random variable vector; $\mathrm{C}$ is the objective function; $\beta_{i}$ is the $i$-th reliability index;
$\beta_{i}^{T}$ is the reliability index limits; $h_{j}$ is the $j$-th deterministic constrain; $m$ is the number of the performance functions; $M$ is the number of the total constrains. The design variable vector could include some independent deterministic variables and take the parameters of probability distribution as variables. The deterministic constrains include variable limits and the drift limits of storey.

Reliability index $\beta_{i}$ could be obtained by the MPFP method. The non-normal random variable $\mathrm{X}$ is converted to independent standard normal random variable $\mathrm{U}$ by Rosenblatt transition. MPFP method can be implemented by the following formula:

$$
\min :\|\mathbf{u}\| \quad \text { s.t. }: G_{i}(\mathbf{X})=0
$$

where, $\mathbf{u}$ is the independent normal variable which is obtained by converting the random variable $\mathrm{X} ; G_{\mathrm{i}}$ is the performance function. The solution $\mathbf{u}^{*}$ of upper formula is the MPFP, and the distance of the MPFP to the original point is reliability index $\beta_{i}=\left\|\mathbf{u}_{i}^{*}\right\|$.

CMAES algorithm. CMAES is a kind of population based random searching evolutionary algorithm. This algorithm has two main features: (1) offspring are generated by sampling according to the multivariate normal distribution; (2) covariance matrix is updated by a new technology. In each iteration operation of CMAES, the step of mutation operator and the elements included in covariance matrix should be updated. Details can be referenced in [15].

Original kriging model. Kriging model is composed of a global regression model and a nonparametric Gaussian random process. The coefficients of covariance matrix are evaluated by maximum likehood estimation. The kriging model is regarded as the most optimal linear unbiased estimation. Details can be referenced in [14].

The weighted summing operations consider these factors: (1) the responses and its distribution in a certain neighbourhood of the sampling points; (2) the positions of the test and sampling points; (3) and the global information of the domain of definition. Hence, for a certain value domain, the more sampling points distributing in, the better response could be get. Meanwhile, CMAES is a population based algorithm whose searching region will gradually decrease along with the iteration. Increasing the sample points in the decrescent searching domain will improve the approximate accuracy of 
kriging model in this most possible domain where optimal solution points could locate.

\section{RBDO-KRIGING ALGORITHM}

Kriging model is used to agent performance function $\mathrm{G}(\mathbf{d}, \mathbf{X})$. The average of the design variable vector $(\mathbf{d}, \mathbf{X})$ includes deterministic design variable vector $\mathbf{d}$ and the mean values of the random variable vector $\mathbf{m x}$.

$$
\mathbf{m n}=[\mathbf{d}, \mathbf{m x}]
$$

refine kriging model. Latin Hypercube Sampling is selected to sample new sampling points. The step length $\sigma$ of CMAES reflects the variable disturbance in iteration. When

$$
\sigma<1 \mathrm{e}-4
$$

The refining process is started. [ld,ud] is determined by following formulas:

$$
\begin{aligned}
& \mathbf{l d}=\mathbf{m}-0.3(\mathbf{u d}-\mathbf{l d}) \\
& \mathbf{u d}=\mathbf{m}+0.3(\mathbf{u d}-\mathbf{l d})
\end{aligned}
$$

$\mathbf{m}$ is corresponding to $\mathbf{u}_{\mathrm{m}}^{*}$ in standard normal distribution $\mathrm{U}$. After the transformation can obtain:

$$
\mathbf{m n}=[\mathbf{m}, \mathbf{m x}]
$$

$\mathbf{m}$ is corresponding to $\mathbf{u}_{\mathrm{m}}^{*}$ in standard normal distribution $\mathrm{U}$. After the transformation, can obtain: $\mathbf{X}^{*}=T^{-1}\left(\mathbf{u}_{\mathrm{m}}^{*}\right)$.

Random variable $\mathbf{X}^{\mathrm{b}}$ corresponding to the optimal solution is in the neighbourhood of $\mathbf{X}^{*}$, the refine region of the random variable [IX,uX] can be defined as:

$$
\begin{aligned}
& \mathbf{I X}=\mathbf{X}^{*}-0.3(\mathbf{u X}-\mathbf{I X}) \\
& \mathbf{u X}=\mathbf{X}^{*}+0.3(\mathbf{u X}-\mathbf{l X})
\end{aligned}
$$

The refine kriging model will be started when formula (4) is meet in the outer loop. The Pseudo of RBDO based on adaptive kriging model is shown in Figure I.

\section{NUMERICAL EXAMPLE}

A 2- bay 10- storey (2b10s) reinforced concrete frame numerical example is used to verify the proposed algorithm, and 5 cases are compared (Figure II). Reliability optimization designs of each case are run 20 times respectively and the best result of these runs are shown in Table I. The structural arrangement of 2b10s RC frame is shown in Figure II. The deterministic design variables are the depths of the beams and columns, whose value range is $[0.30 \mathrm{~m}, 0.90 \mathrm{~m}]$. The random variables are the base shear force. The variable coefficient of the section is 0.05 , and the distribution type is lognormal. The mean value of the shear force is $371.34 \mathrm{kN}$, and its variable coefficient is 0.1 , the distribution type is extreme value type I distribution. The reliability index for constrain response displacements less than its limits of each storey is set to 1.5[16].

Function:d* $=$ RBDO-kriging (a,b)

Load the feasible region $[\mathbf{a}, \mathbf{b}]$ of $\mathbf{d}$;

Determine the optimization parameters in the outer loop. //S is the dimensions of the design variables //

Population: $\mathrm{N} 2=2 \mathrm{~S}$; the maximal number of function evaluation: maxfeval $2=200 \mathrm{~S}$;

Initialize CMAES parameters.

$\mathbf{m}=\mathbf{a}+\operatorname{rand}(1, \mathrm{~S}) *(\mathbf{b}-\mathbf{a}) ; / /$ rand is the random function//

$\mathbf{l d}=\mathbf{a} ; \mathbf{u d}=\mathbf{b} ; \mathbf{I X}=\mathbf{m n}-\mathbf{5} \mathbf{s t} ; \mathbf{u X}=\mathbf{m n}+\mathbf{5 s t} ; \mathbf{l b}=[\mathbf{a}, \mathbf{I X}] ; \mathbf{u b}=[\mathbf{b}, \mathbf{u X}] ; t=f=0$;

$/ / \mathbf{m n}$ and st are mean value and the variance of $\mathrm{X}$ respectively//

Train kriging model to get new kriging and TrainSet;

while $t<\operatorname{maxFE}$

update d; $\mathrm{y}_{1}=0 ; \mathrm{y}_{2}=0$;

for $\mathrm{i}=1: \mathrm{N} 2 \quad / / \mathrm{M}$ is the number of the reliability constrains//

for $\mathrm{j}=1: \mathrm{M}$; compute $\beta$; predict structural responses; $\mathrm{y}_{1}=\mathrm{y}_{1}+\max \left(0, \beta^{\mathrm{T}} / \beta-1\right) ; \mathrm{y}_{2}=\mathrm{y}_{2}+\max \left(0, \mathrm{~h} / \mathrm{h}^{\mathrm{T}}-1\right)$;

end for; $\mathrm{y}_{\mathrm{i}}=C(\mathbf{d}) *\left(1+\mathrm{y}_{1}+\mathrm{y}_{2}\right)^{2}$;

end for; $\quad$ set best $\mathbf{d}_{i}$ is $\mathbf{d}^{*}$; update $\mathbf{m} 、 \mathbf{P}_{c} 、 \mathbf{C} 、 \mathbf{P}_{\sigma}$ and $\sigma$;

If $\sigma<1 \mathrm{e}-4$

$\mathrm{X}^{*}=T^{-1}($ Reliability (m,mn,st,kriging)); // Reliability ( ) means computing reliability index//

$\mathbf{l d}=\mathbf{m}-0.3(\mathbf{u d}-\mathbf{l d}) ; \mathbf{u d}=\mathbf{m}+0.3(\mathbf{u d}+\mathbf{l d}) ; \mathbf{l X}=\mathbf{X}^{*}-0.3(\mathbf{u X}-\mathbf{I X}) ; \mathbf{u X}=\mathbf{X}^{*}+0.3(\mathbf{u X}-\mathbf{I X}) ; \mathbf{l b}=[\mathbf{a}, \mathbf{l X}] ; \mathbf{u b}=[\mathbf{b}, \mathbf{u X}] ;$

$[\mathbf{l b}, \mathbf{u b}]=$ inboundary $([\mathbf{a}, \mathbf{m n}-5 \mathbf{s t}],[\mathbf{b}, \mathbf{m n}+5 \mathbf{s t}]) ;[$ kriging,TrainSet $]=$ Reifine $(\mathbf{l b}, \mathbf{u b}$, TrainSet $) ; \sigma=0.3(\mathbf{u b}-\mathbf{l b}) ; t=t+\mathrm{S} ;$

end if

if $\max (\mathbf{D})>1 \mathrm{e} 7 \min (\mathbf{D})$ OR $f>\operatorname{maxfeval2}$; break; end if; $f=f+\mathrm{N} 2$;

end while; 


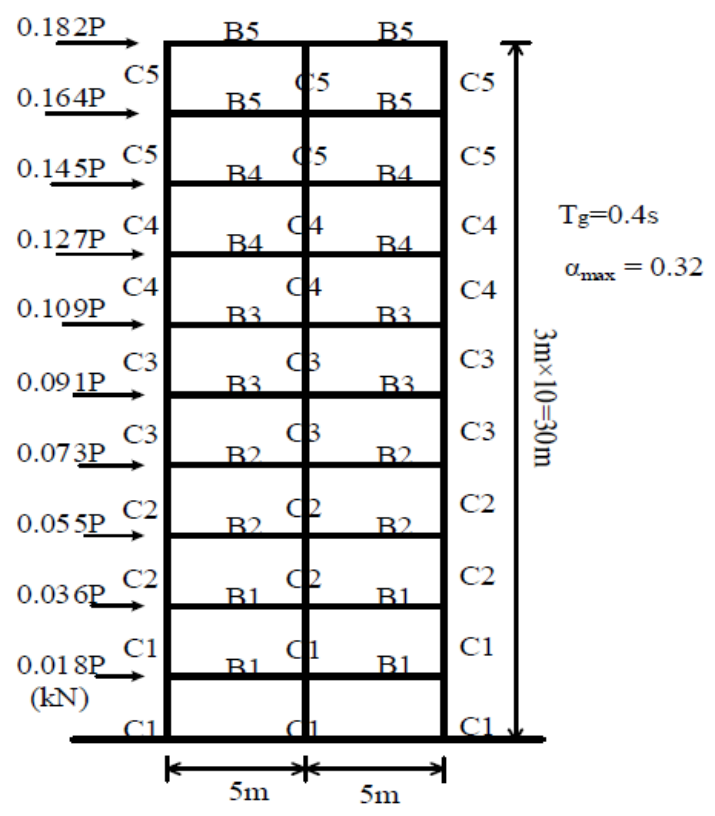

- case1: deterministic optimization without reliability constrains;

- case2: RBDO based on RBDO-kriging algorithm with reliability constrains;

- case3: RBDO based on original kriging model with reliability constrains;

- case4: RBDO based on PSO algorithm where PSO is used in the outer loop, and structural responses are directly computed without agent model;

- case5: RBDO based on DE algorithm where DE is used in the outer loop, and structural responses are directly computed without agent model.

FIGURE II. THE STRUCTURE ARRANGEMENT OF 2B10S RC FRAME

TABLE I. STATISTICS OF OBJECTIVE FUNCTION

\begin{tabular}{ccc|ccc|cccc|ccccc}
\hline \multicolumn{3}{c|}{ case1(RMB) } & \multicolumn{3}{c|}{ case2(RMB) } & \multicolumn{3}{c|}{ case3(RMB) } & \multicolumn{3}{c}{ case4(RMB) } & \multicolumn{2}{c}{ case5(RMB) } \\
\hline optimal & mean & worst & optimal & mean & worst & optimal & mean & worst & optimal & mean & worst & optimal & mean & worst \\
\hline 3778 & 3778 & 3778 & 5483 & 5484 & 5486 & 5614 & 5620 & 5631 & 6042 & 6456 & 7013 & 6044 & 6320 & 6589 \\
\hline
\end{tabular}

TABLE II. CENTERS OF REFINING REGION FOR 2C10S RC FRAME

\begin{tabular}{cccccccccccc}
\hline section & C1h & C2h & C3h & C4h & C5h & B1h & B2h & B3h & B4h & B5h \\
\hline The first time & 0.821 & 0.541 & 0.501 & 0.531 & 0.521 & 0.900 & 0.819 & 0.681 & 0.656 & 0.560 \\
the second time & 0.812 & 0.533 & 0.484 & 0.523 & 0.506 & 0.900 & 0.830 & 0.710 & 0.698 & 0.571 \\
optimal & 0.797 & 0.527 & 0.480 & 0.507 & 0.497 & 0.900 & 0.827 & 0.705 & 0.686 & 0.615 \\
\hline
\end{tabular}

TABLE III. RESULTS OF OPTIMAL DESIGN FOR 2B4S RC FRAME

\begin{tabular}{|c|c|c|c|c|c|c|c|c|c|c|c|}
\hline section & case1 & case2 & case3 & case4 & case5 & section & case1 & case2 & case3 & case4 & case5 \\
\hline C1h & 0.602 & 0.797 & 0.811 & 0.835 & 0.837 & B1h & 0.899 & 0.900 & 0.900 & 0.900 & 0.900 \\
\hline $\mathrm{C} 2 \mathrm{~h}$ & 0.415 & 0.527 & 0.534 & 0.553 & 0.554 & B2h & 0.815 & 0.827 & 0.831 & 0.871 & 0.864 \\
\hline C3h & 0.405 & 0.480 & 0.485 & 0.522 & 0.524 & B3h & 0.817 & 0.705 & 0.714 & 0.722 & 0.721 \\
\hline C4h & 0.383 & 0.507 & 0.523 & 0.510 & 0.513 & B4h & 0.670 & 0.686 & 0.699 & 0.687 & 0.687 \\
\hline C5h & 0.354 & 0.497 & 0.520 & 0.607 & 0.601 & B5h & 0.427 & 0.615 & 0.563 & 0.500 & 0.500 \\
\hline
\end{tabular}

As shown in Table I, the statistics of case 1 and case 2 are more stable, while due to lightly poor agent performance on high dimension functions of kriging model, the statistics of case 3 is less stable. Meanwhile the stability of the statistics of case 4 and case 5 are worst.

The optimization results of 5 cases are shown in Table II: the sections obtained form case 1 is minimal, and that obtained from case 4 and case 5 are maximal. The adoption of the reliability constrains resulted in the fact that the sizes of $\mathrm{C} 4 \mathrm{~h}$ and C5h are larger than C3h's. If storey displacements obeyed an unknown distribution, the reliability indexes of storey displacements are related to the value of random variable. As a result, the differences between of the results in deterministic and random optimization are mainly because of reliability constrains.

The convergence processes of the cost of 5 cases are shown in Figure III. The costs of 5 cases are converged to 3778, 5483, 5614, 6042 and 6044RMB respectively. In the RBDO cases, case 2 have the best convergence. Noted that the refine of the kriging model give rise of the waviness and long duration of the convergence. In case 2, RBDO-kriging algorithm invoked the kriging refining operator in the 58-th and 157-th iteration step, and the central point at these two steps are shown in Table III. It can be seen that after the refining operator, the step 
length is updated, which increased the waviness of the convergence but improved the effectiveness of the algorithm.

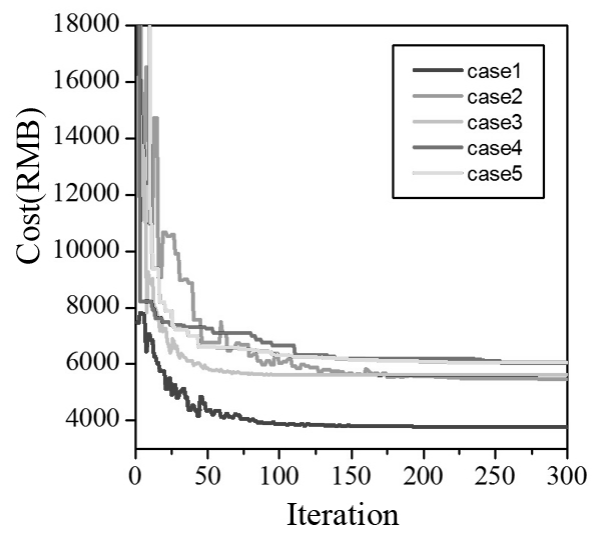

FIGURE III. COST CONVERGENCE FOR 2B 10S RC FRAME

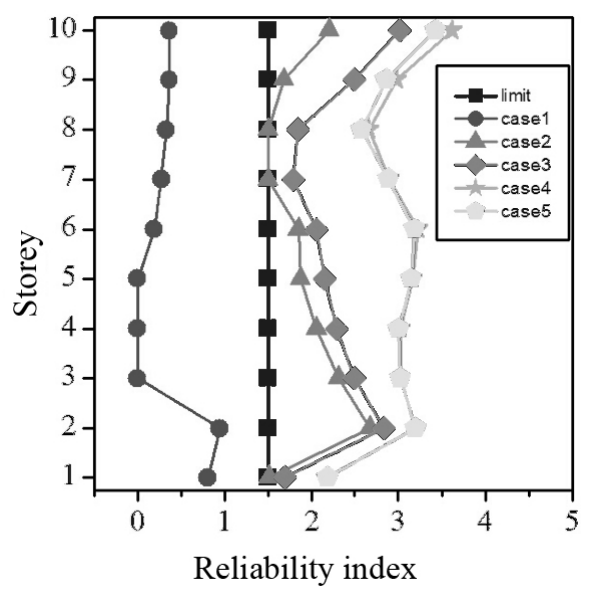

FIGURE IV.

RELIABILITY INDEX OF OPTIMAL DESIGN FOR 2B4S RC FRAME

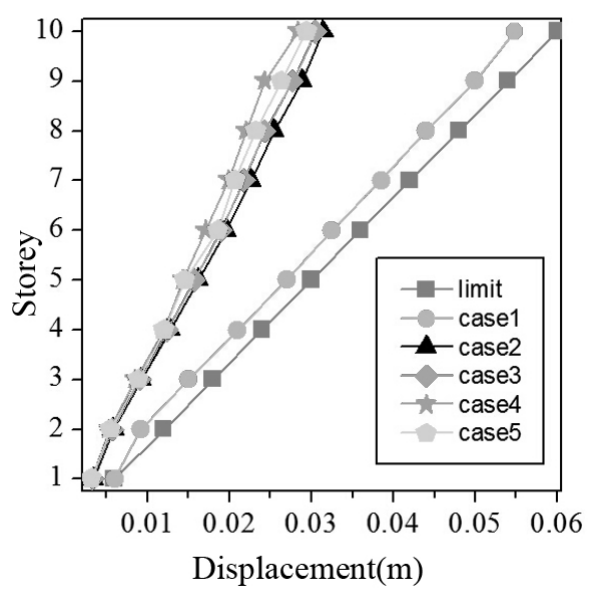

FIGURE V.

DISPLACEMENT FOR 2B10S RC FRAME

The numbers of structural analysis of 5 cases are shown in Table IV, that of case 2 are minimal. Considered Figure III simultaneously, in 4 RBDO algorithms the proposed one stably and quickly converged to the best solution with the minimum number of the structural analysis.

TABLE IV. NUMBER OF FINITE ELEMENT ANALYSIS

\begin{tabular}{ccccc}
\hline case & case2 & case3 & case4 & case5 \\
\hline optimal cost(RMB) & 5483 & 5614 & 6042 & 6044 \\
structural analysis times & 1356 & 3000 & 9000 & 9000 \\
\hline
\end{tabular}

The reliability indexes corresponding to the optimal solution are shown in Figure IV: the reliability indexes of each storey are less the limit 1.5 in case1, and case 2 have the minimal indexes. It is because that the optimization design of case 1 abandoned the reliability constrains. The kriging refine strategy facilitated the kriging model to be with a high agent performance within the optimal region. Meanwhile the poor agent precision of original kriging model within the global define region in case 3 resulted in deviation of its result from the optimal solution. The case 4 and case 5 without agent model needed a huge number of structural analysis times as the reliability computing directly on the evaluation of the performance function.

The displacements of 5 cases are shown in Figure V: the displacement of case 2 is larger than case 3 , case 4 and case 5 . It is shown that the use of the reliability constrains make a more reliable design, which led to the stiffness of the structure on the horizontal direction larger than that of the deterministic optimization design results.

\section{CONCLUSION}

In this paper a double loop framework RBDO algorithm based on an adaptive kriging model is proposed. The refine kriging model for the RBDO problem is built, hence a common kriging model that could be used in the inner and outer loop both is obtained. The following important conclusions are verified by a numerical simulation:

- The efficiency of the proposed kriging-RBDO in this paper is high. The comparison of 5 cases show that kriging-RBDO can obtain the best solution with minimal structural times.

- $\quad$ The refined kriging model is effective. In the searching process of CMAES, refine kriging model can determined the design variables and the refine region of the random variables accurately, and within this refine region kriging model could be automatically updated to guarantee the searching ability of CMAES.

- The deterministic optimizations could obtain the solution with the minimal cost but the maximum displacement. Meanwhile, the reliability indexes of these deterministic optimizations are minimal but could not meet the reliability requirement.

\section{REFERENCES}

[1] Beck A T, Gomes W J D S, "A comparison of deterministic, reliabilitybased and risk-based structural optimization under uncertainty," Probabilistic Engineering Mechanics. Vol. 28, pp. 18-29, 2012. 
[2] Min Zheng, Suguo Liang, Tiehua Xiong, "Study on the reliability-based wind resistant optimization of transmission line towers," Journal of Hunan University, vol. 6, pp. 35-42, 2014.

[3] Zhen Sun, Weiqing Liu, Shuguang Wang, etc, "Parametric optimization of a base-isolated structure based on system reliability," Journal of vibration and shock, vol. 32, pp. 6-10, 2013.

[4] Enevoldsen I, Sorensen JD, "Reliability-based optimization in structural engineering”, Structural Safety, vol. 15, pp. 169-196, 1994.

[5] Du X, Chen W, "Sequential optimization and reliability assessment method for efficient probabilistic design,” Journal of Mechanical Design, vol. 126, pp. 225-233, 2004.

[6] Shan S, Wang G, "Reliable design space and complete single-loop reliability-based design optimization," reliability engineering \& system safety, vol. 93, pp. 1218-1230, 2008.

[7] Cho H, Bae S, Choi K K, et al, "An efficient variable screening method for effective surrogate models for reliability-based design optimization," Structural and Multidisciplinary Optimization, pp. 1-22, 2014.

[8] Aoues Y, Chateauneuf A, "Benchmark study of numerical methods for reliability-based design optimization," Structural and multidisciplinary optimization, vol. 41, pp. 277-294, 2010.

[9] Dubourg V, Sudret B, Bourinet J, "Reliability-based design optimization using kriging surrogates and subset simulation,” Structural and Multidisciplinary Optimization, vol. 44, pp. 673-690, 2011.

[10] Kim C, Choi K K, "Reliability-based design optimization using response surface method with prediction interval estimation," Journal of Mechanical Design, vol. 130 pp. 1-12 2008.

[11] Li H, "Reliability-based design optimization via high order response surface method," Journal of Mechanical Science and Technology, vol. 27, pp. 1021-1029, 2013.

[12] Sakata S, Ashida F, "Ns-kriging based microstructural optimization applied to minimizing stochastic variation of homogenized elasticity of fiber reinforced composites," Structural and Multidisciplinary Optimization, vol. 38, pp. 443-453, 2009.

[13] Forrester A I, Keane A J, "Recent advances in surrogate-based optimization,” Progress in Aerospace Sciences, vol. 45, pp. 50-79, 2009.

[14] Cressie N, “The origins of kriging," Mathematical Geology, vol. 22, pp. 239-252, 1990.

[15] Auger A, Hansen N, "A restart CMA evolution strategy with increasing population size," The 2005 IEEE Congress on Evolutionary Computation, Edinburgh: IEEE, pp. 1769-1776, 2005.

[16] Ministry of Housing and Urban-Rural Development of the People's Republic of China, "Unified standard for reliability design of building structures (GB 50068-2001),” Peking: China Architecture \& Building Press, 2002. 